\title{
IS THERE AN OBLIQUE MAGNETIC ROTATOR INSIDE THE SUN?*
}

(Invited Review, Abstract)

\author{
G. R. ISAAK
}

Department of Physics, University of Birmingham, Birmingham, B15 2TT, UK

\begin{abstract}
The size of the rotational splitting recently observed (Claverie et al., 1981) is correlated with the $12.2^{\mathrm{d}}$ variation in the measurements of solar oblateness observed by Dicke (1976) and implies a convection zone of depth of $0.1 R_{\odot}$. The near equality of amplitudes of global velocity oscillations (Claverie et al., 1981) of the various $m$ components of the $l=1$ and $l=2$ modes as seen from the Earth viewing the Sun nearly along the equator is unexpected for pure rotational splitting. It is suggested that a magnetic perturbation is present and an oblique asymmetric magnetic rotator with magnetic fields of a few million gauss is responsible. A more detailed account was submitted to Nature.
\end{abstract}

\section{References}

Claverie, A., Isaak, G. R., McLeod, C. P., van der Raay, H. B., and Roca Cortes, T.: 1981, Nature 292, 443. Dicke, R. H.: Solar Phys. 47, 475.

* Proceedings of the 66th IAU Colloquium: Problems in Solar and Stellar Oscillations, held at the Crimean Astrophysical Observatory, U.S.S.R., 1-5 September, 1981. 